\title{
Perfil Lipídico de Jovens Escolares entre 2 e 19 anos no Interior da Bahia
}

\author{
Sara Costa Sampaio ${ }^{1}$; Beatriz Rocha Sousa ${ }^{2}$; Diana Cirqueira Oliveira ${ }^{3}$; \\ Adna Gorette Ferreira Andrade ${ }^{4}$; Stênio Fernando Pimentel Duarte ${ }^{5}$;
}

Resumo: A presente pesquisa teve como objetivo descrever o perfil lipídico em crianças e adolescentes com idade entre 2 e 19 anos em escolares no interior da Bahia, identificando fatores associados a essas alterações que podem levar a complicações na idade adulta. Métodos: Os participantes foram submetidos a avaliação antropométrica, foram coletadas amostras de sangue após jejum de 12 horas, e as seguintes avaliações foram realizadas por métodos enzimáticos: níveis séricos de colesterol total, colesterol LDL, triglicerídeos e VLDL. As informações relacionadas a consumo alimentar, atividade física e condições socioeconômicas e de moradia da família foram fornecidas pelos responsáveis durante a avaliação através de um questionário on-line. Os dados foram analisados com o programa estatístico SPSS 22.0 que inclui o test-t de Student e o teste exato de Fisher.Resultados: Das 97 crianças e adolescentes analisados no presente estudo, cerca de $30 \%$ apresentaram um perfil lipídico aterogênico, caracterizado por altos níveis de triglicerídeo, VLDL colesterol total e colesterol LDL. A prevalência de obesos foi $28,95 \%$. Os meninos apresentaram níveis de triglicerídeo, VLDL, colesterol total e LDL mais elevados do que as meninas. Observou-se associação positiva e significante entre dislipidemia e excesso de peso, consumo excessivo de alimentos de risco como lipídios e carboidratos.Crianças e adolescentes apresentaram os mesmos valores de lipídios no sangue, o que não é esperado para crianças nessa fase do desenvolvimento.Conclusão: Na presente população, uma grande parte apresentou um perfil lipídico aceitável, o que mostra que mesmo com alto consumo de alimentos considerados aterogênicos isoladamente não determina uma alteração significativa nos níveis lipídicos, porém a associação de diversos fatores como obesidade, sedentarismo, alimentação inadequada e principalmente fator genético poderá levar a umadislipidemia, o que supostamente pode estar representado pela pequena parcela a qual apresentou um perfil lipídico desfavorável e que devemos redobrar a atenção objetivando a prevenção de doenças cardiovasculares e obesidade precocemente.

Palavras-chave: Dislipidemia. Sobrepeso. Aterosclerose.

\section{Lipid Profile of Young Schoolchildren between two and nineteen years old in the State of Bahia}

\begin{abstract}
This study aimed to describe the lipid profile in children and adolescents aged 2 to 19 years in schoolchildren in the interior of Bahia, identifying factors associated with these alterations that may lead to complications in adulthood. Methods: Participants underwent an anthropometric evaluation, blood samples were collected after a 12-hour fast, and the following evaluations were performed by enzymatic methods: serum levels of total cholesterol, LDL cholesterol, triglycerides and VLDL. Information related to food consumption, physical activity, and socioeconomic and family housing conditions were provided by those responsible during the evaluation through an online questionnaire. Data were analyzed using the SPSS 23.0 statistical program, which included Student's t-test and Fisher's exact test. Results: Of the 97 children and adolescents analyzed in the present study, about $30 \%$ presented an atherogenic lipid profile, characterized by high triglyceride levels, VLDL total cholesterol and LDL cholesterol. The prevalence of obese individuals was $28.95 \%$. Boys had higher triglyceride, VLDL, total cholesterol and LDL levels than girls. A positive and significant association was observed between dyslipidemia and overweight, excessive consumption of high-risk foods such as lipids and carbohydrates. Children and adolescents presented the same values of blood lipids, which is not expected for children at this stage of
\end{abstract}

\footnotetext{
${ }^{1}$ Graduanda em Farmácia pela Faculdade Independente do Nordeste - FAINOR. E-mail: sarasampaio27@yahoo.com.br

${ }^{2}$ Graduanda em Nutrição pela Faculdade Tecnologia e Ciências - FTC. E-mail: beatrizrsousa@ outlook.com

${ }^{3}$ Graduanda em Farmácia pela Faculdade Independente do Nordeste - FAINOR. E-mail: daicirqueira@ hotmail.com

${ }^{4}$ Graduada em Fisioterapia pela Faculdade Tecnologia e Ciência - FTC, especialização em Saúde Pública, atualmente docente.

${ }^{5}$ Graduação em Ciências Biológica, Mestrado e Doutorado em Fisiopatologia Clínica e Experimental pela Universidade do Estado do Rio de Janeiro - UERJ, atualmente é docente da Faculdade Independente do Nordeste e Faculdade Tecnologia e Ciências, Vitória da Conquista/BA. E-mail: steniofernando@gmail.com
} 
development. Conclusion : In the present population, a large proportion presented an acceptable lipid profile, which shows that even with high consumption of food considered atherogenic alone does not determine a significant change in lipid levels, but the association of several factors such as obesity, sedentary lifestyle, inadequate diet and Mainly genetic factor may lead to dyslipidemia, which may be represented by the small portion which presented an unfavorable lipid profile and that we should redouble our attention aiming at the prevention of cardiovascular diseases and obesity early.

Keywords:Dyslipidemia. Overweight. Atherosclerosis

\section{Introdução}

A dislipidemia é uma alteração no metabolismo das gorduras, levando a concentração anormal de lipídeos na corrente sanguínea, e pode ser estabelecida por fatores ambientais ou genéticos, porém o principal fator para o aumento do colesterol é a ingestão alimentar caracterizada pelo excesso de ácidos graxos saturados, colesterol e energiatotal(SERVICES et al., 2012). A classificação ambiental pode ser determinada por meios de análises bioquímicas que considera os valores de CT (colesterol total), TG (triglicerídeos), LDL-c (lowdensitycholesterol) e HDL (high densitycholesterol)(NOGUEIRA; CRISTINA CORRÊA DE SOUZA, 2014).

Níveis séricos alterados desses lipídios em crianças podem evoluir, mantendo mesmos níveis de colesterol até a idade adulta, levando a agravos como aterosclerose, que é uma doença inflamatória crônica caracterizada pela formação de placas de lipídios dentrodos vasos

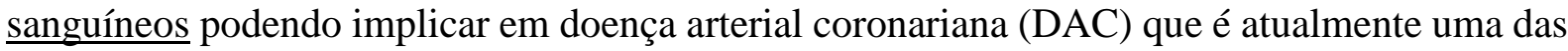
principais causas de morbimortalidade no Brasil e no mundo, decorrente da combinação de vários fatores, podendo ter início sem sinais e sintomas na infância, com desenvolvimento gradual durante a adolescência e a idade adulta(BRASILEIRA; CARDIOLOGIA, 2013). Apenas nos últimos anos, a dosagem de colesterol durante a infância tem recebido maior atenção, por isso, é de grande interesse clínico a identificação de crianças com fatores de risco para aterosclerose permitindo intervenções precoces, diminuindo o processo aterosclerótico e consequentemente prevenindo ou reduzindo a ocorrência de doençascardiovasculares (EILAND LS et al., 2010). 
Já que vêm sendo destacado que as mudanças de hábitos alimentares e estilo de vida estão associados a uma tendência crescente da prevalência da dislipidemia podemos minimizar esses riscos e evitar esses eventos, adotando medidas de extrema importância já na infância em relação a preferências alimentares, que se forem introduzidos nesse período, podem se tornarpermanentes(RAMOS et al., 2011).

A condição alimentar da criança e do adolescente é relevante, pois a presença de obesidade nesta faixa etária tem sido associada ao surgimento prematuro de hipertensão arterial, dislipidemias, aumento da ocorrência de diabetes tipo 2(ENES; SLATER, 2010).

O crescimento do sobrepeso e obesidade em idades cada vez mais antecipadas tem motivado uma atenção ainda maior de pesquisadores e profissionais da área de saúde, em virtude dos danos e agravos à saúde ocasionados pelo peso em excesso, como hipertensão arterial, cardiopatias, diabetes, hiperlipidemias, dentreoutras(SPOSITO et al., 2007). A obesidade tem sido relatada como um problema significativo de saúde pública da atualidade e vem sendo evidente no cenário epidemiológicomundial, onde mudanças demográficas, socioeconômicas e epidemiológicas levaram a uma alteração dos padrões nutricionais, aumentando significativamente a prevalência desobrepeso(UREK; CRNCEVIĆ-UREK; CUBRILO-TUREK, 2007).

Sua prevalência expandiu nas últimas décadas em todo o mundo, até mesmo nos países em desenvolvimento, como o Brasil, onde antigamente predominavam os problemas referentes àdesnutrição (KELSEY et al., 2014).

Sendo assim, o objetivo desse estudo foi verificar a ocorrência de alterações no perfil lipídico de jovens com idade entre 2 e 19 anos, estudando fatores ambientais relacionados com estilo de vida, entre os quais estão incluídas mudanças negativas nos hábitos alimentares, obesidade e sedentarismo que podem estar relacionados com aumento dos níveis lipídicos. Para determinação desse perfil lipídico foram aplicados questionários socioeconômicos e questionários de frequiência alimentar, seguido de coleta de sangue para determinação de concentrações lipídicas e por fim, realizado a coleta de medidas antropométricas. 


\section{Metodologia}

O trabalho possui um delineamento epidemiológico transversal, de caráter retrospectivo e descritivo e abordagem quantitativa, a pesquisa foi desenvolvida entre os meses de junho de 2016 até maio de 2017 e a coleta de dados decorreu entre os meses de junho a dezembro de 2016, no município de Vitória da Conquista, localizado no interior da Bahia onde possui aproximadamente 306.866 habitantes e está há $509 \mathrm{~km}$ de distância da capital Salvador, apresenta coordenada geográfica como latitude $-14^{\circ} 53^{\prime}$ e longitude-40 $48^{\prime}$ (IBGE, 2013).

A população estudada foi de 97crianças e adolescentes entre 2-19 anos, de ambos os sexos escolhidos aleatoriamente, no qual, os dados coletados foram fornecidos pelos responsáveis através de questionários on-line com as informações relacionadas a consumo alimentar, atividade física, condições socioeconômicas e de moradia da família. Os participantes foram submetidos à avaliação antropométrica, foram coletadas amostras de sangue após jejum de 12 horas, e as seguintes avaliações foram realizadas por métodos enzimáticos: níveis séricos de colesterol total, colesterol LDL, triglicerídeos e VLDL.

Todos os participantes foram informados a respeito dos objetivos do estudo e motivo da pesquisa, sendo garantido durante o questionário o sigilo e anonimato aos participantes, através da assinatura do Termo de Consentimento Livre e Esclarecido (TCLE), em duas vias, que continha dados pertinentes aos aspectos éticos e vigentes dispostos na resolução $n^{\circ} 466 \backslash 12$ do Conselho Nacional de Saúde (CNS). O questionário foi respondido on-line em uma sala com computadores em tempo aproximado de 20 minutos e as respostas obtidas foram tabuladas por meio do programa estatístico SPSS 22 IBM® e também delineados pelo teste qui-quadrado de Pearson para comparar as frequências das variáveis analisadas.

A pesquisa foi aprovada pelo Comitê de Ética da Faculdade Independente do Nordeste - FAINOR, e convergiu com os preceitos éticos dispostos na Resolução nº 466/12 do Conselho Nacional de Saúde (CNS). 
Id on Line Revista Multidisciplinar e de Psicologia

Id on Line Multidisciplinary and Psycology Journal

\section{Resultados}

A caracterização da amostra e a comparação entre meninos, meninas em relação às variáveis idade, IMC, atividade física, dieta, colesterol total, LDL, VLDL, triglicérides e consumo de lipídios e carboidratos são apresentadas na tabela 1. Das 97 crianças e adolescentes, 56 ( $57,7 \%)$ eram meninas e $41(42,3 \%)$ eram meninos. Os níveis lipídicos médioscomo colesterol total, LDL, VLDL e triglicerídeos foram significativamente mais elevados nos meninos do que nas meninas. De acordo com os valores do IMC, obtivemos amostras com todas as idades, porém, a amostra em maior quantidade foi representada por jovens com 19 anos (Gráfico1),a análise revelouque 28,95\% das crianças e adolescentes eram obesas e a inatividade física estava presente em 61,11\% deles ( 24 meninas e 9 meninos) e apenas $16 \%$ faziam dieta sendo 10 meninas e 6 meninos.

Tabela 1- Comparação entre meninos, meninas em relação à variáveis idade, IMC, atividade física, dieta, colesterol total, LDL, VLDL, triglicérides e consumo de lipídios e carboidratos

\begin{tabular}{|c|c|c|c|c|c|c|c|}
\hline \multirow{2}{*}{\multicolumn{2}{|c|}{ Variáveis }} & \multicolumn{2}{|c|}{$\begin{array}{c}\text { População Geral } \\
\text { (PG) }\end{array}$} & \multicolumn{2}{|c|}{$\begin{array}{c}\text { População } \\
\text { Feminina (PF) }\end{array}$} & \multicolumn{2}{|c|}{$\begin{array}{c}\text { População Masculina } \\
\text { (PM) }\end{array}$} \\
\hline & & $\mathrm{N}$ & $\%$ & $\mathrm{~N}$ & $\%$ & $\mathrm{~N}$ & $\%$ \\
\hline \multicolumn{8}{|l|}{ Idade } \\
\hline & 2-4 Anos & 3 & 3,09 & 1 & 1,8 & 2 & 4,8 \\
\hline & 5-12 Anos & 38 & 39,18 & 18 & 32,3 & 20 & 48,7 \\
\hline & 13 - 19 Anos & 56 & 57,73 & 37 & 65,9 & 19 & 46,5 \\
\hline & Total & 97 & 100 & 56 & 100 & 41 & 100 \\
\hline \multicolumn{8}{|l|}{ IMC } \\
\hline & Obeso & 22 & 28,95 & 8 & 19,51 & 14 & 40 \\
\hline & Não obeso & 54 & 71,05 & 33 & 80,49 & 21 & 60 \\
\hline & Total & 76 & 100 & 41 & 100 & 35 & 100 \\
\hline \multicolumn{8}{|l|}{ Atividade Física } \\
\hline & Ativo & 21 & 38,89 & 12 & 33,33 & 9 & 50 \\
\hline & Não Ativo & 33 & 61,11 & 24 & 66,67 & 9 & 50 \\
\hline & Total & 54 & 100 & 36 & 100 & 18 & 100 \\
\hline \multicolumn{8}{|l|}{ Dieta } \\
\hline & Não & 81 & 83,51 & 46 & 82,15 & 35 & 85,37 \\
\hline & Sim & 16 & 16,49 & 10 & 17,85 & 6 & 14,63 \\
\hline & Total & 97 & 100 & 56 & 100 & 41 & 100 \\
\hline \multicolumn{8}{|l|}{ Colesterol Total } \\
\hline & Normal & 43 & 56,58 & 27 & 65,85 & 16 & 45,71 \\
\hline & Alterado & 33 & 43,42 & 14 & 34,14 & 19 & 54,29 \\
\hline & Total & 76 & 100 & 41 & 100 & 35 & 100 \\
\hline
\end{tabular}


Id on Line Revista Multidisciplinar e de Psicoloqia

Id on Line Multidisciplinary and Psycology Journal

\begin{tabular}{lrrccccc} 
Colesterol - LDL & & & & & & \\
& Normal & 54 & 72,97 & 31 & 77,5 & 23 & 67,65 \\
& Alterado & 20 & 27,02 & 9 & 22,5 & 11 & 32,35 \\
& Total & 74 & 100 & 40 & 100 & 34 & 100 \\
\hline Colesterol - VLDL & & & & & & & \\
& Normal & 57 & 77,03 & 33 & 82,5 & 24 & 70,59 \\
& Alterado & 17 & 22,97 & 7 & 17,5 & 10 & 29,41 \\
& Total & 74 & 100 & 40 & 100 & 34 & 100 \\
\hline Triglicérides & & & & & & & \\
& Normal & 52 & 70,27 & 31 & 77,5 & 21 & 61,76 \\
& Alterado & 22 & 29,73 & 9 & 22,5 & 13 & 38.24 \\
& Total & 74 & 100 & 40 & 100 & 34 & 100 \\
\hline Lipídeos & & & & & & & \\
& Deficiente & 7 & 8,34 & 4 & 7,84 & 3 & 9,37 \\
& Excessivo & 76 & 91,57 & 47 & 92,16 & 29 & 90,63 \\
& Total & 83 & 100 & 51 & 100 & 32 & 100 \\
\hline Carboidrato & & & & & & & \\
& Excessivo & 82 & 100 & 50 & 100 & 32 & 100 \\
& Total & 82 & 100 & 50 & 100 & 32 & 100 \\
\hline
\end{tabular}

Fonte: Pesquisa própria

Gráfico 1- IMC representado por uma amostra em maior quantidade de jovens com 19 anos

\section{Índice de Massa Corporal}

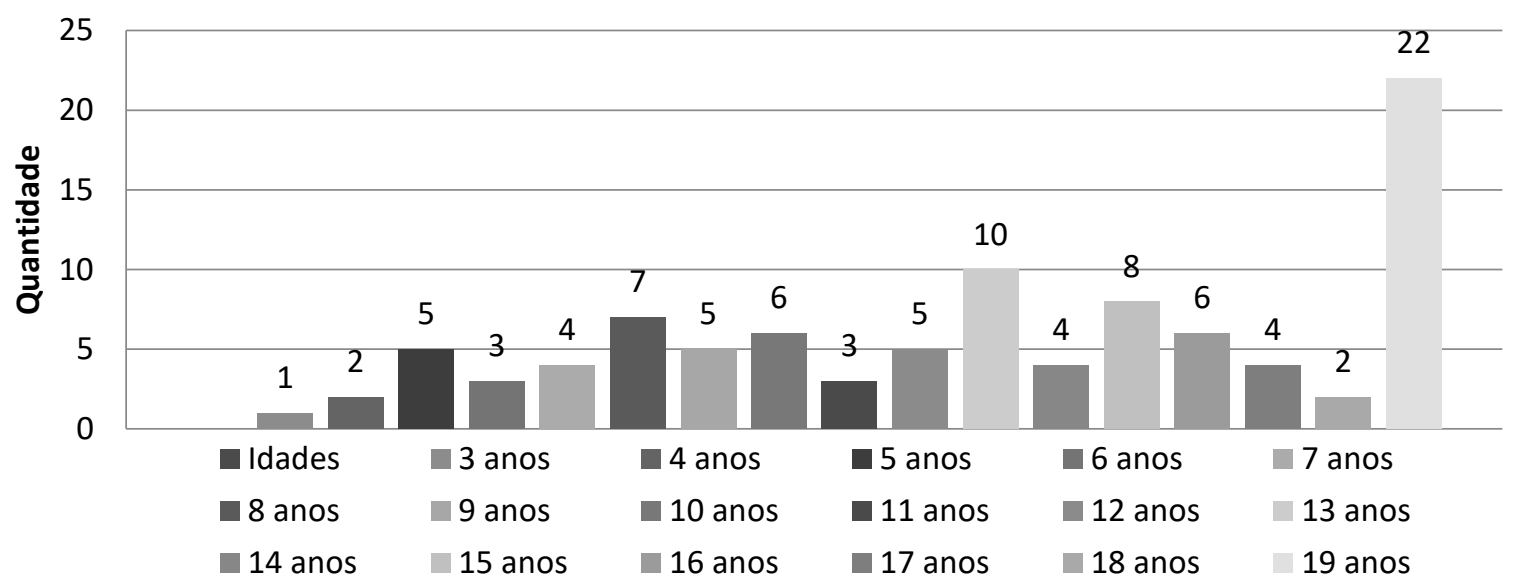

Fonte: Pesquisa própria,2016.

Mais de 70\% de todos os sujeitos do estudo apresentaram um perfil lipídico aceitável, caracterizados por níveis mais elevados de colesterol HDL, níveis mais baixos de colesterol 
LDL, e níveis normais de triglicerídeos e VLDL, que podem ser observados no gráfico 2 e 3.Porém,verificou-se também que houveram alterações nos níveis plasmáticos de colesterol total, Colesterol LDL, VLDL e triglicérides em uma pequena parte dessa população jovem, que representa um percentual respectivamente de 43,42\%,27,02\%, 22,97\% e 29,73\%. Uma parcela pequena mas não menos importante e que devemos ter atenção

Gráfico2- Quantidade de crianças e adolescentes em relação aos níveis de colesterol total, LDL e VLDL

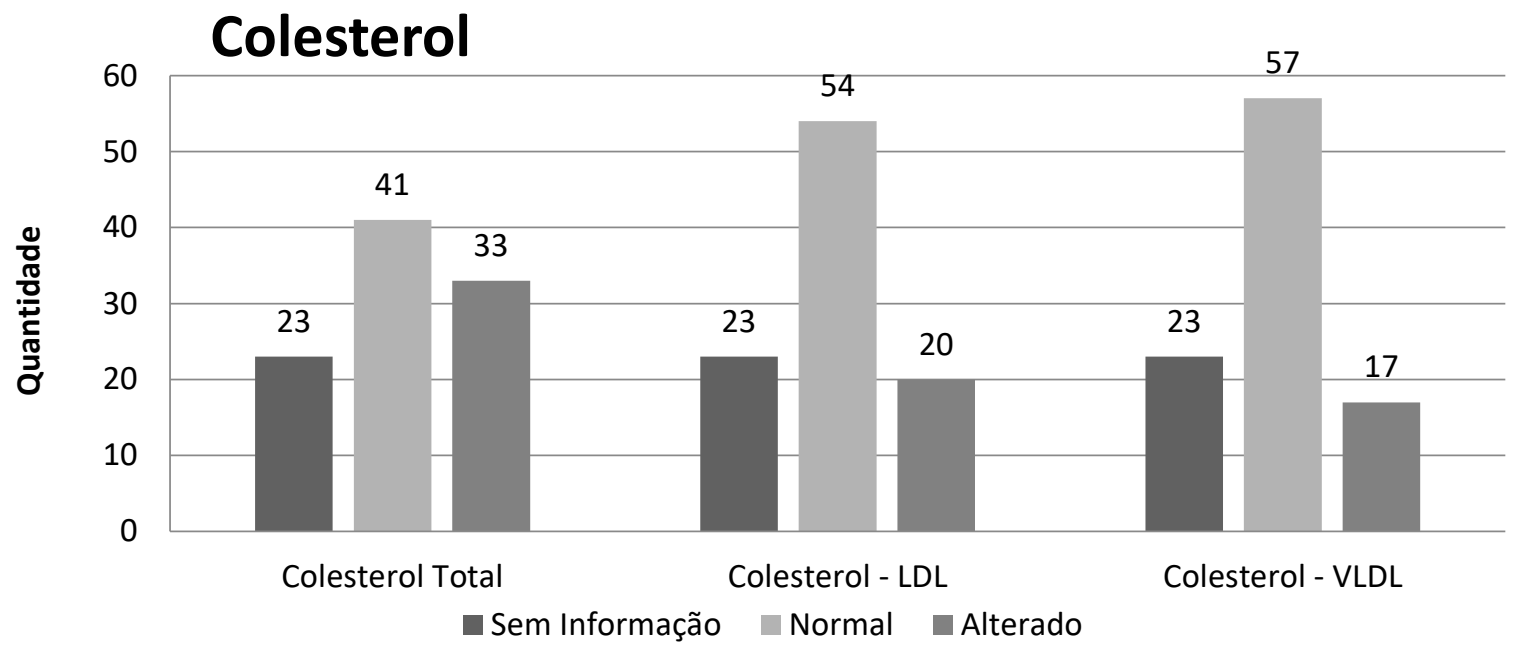

Fonte: Pesquisa própria, 2016.

Gráfico 3- Quantidade de crianças e adolescentes com níveis de triglicérides normais ou alterados

Triglicérides

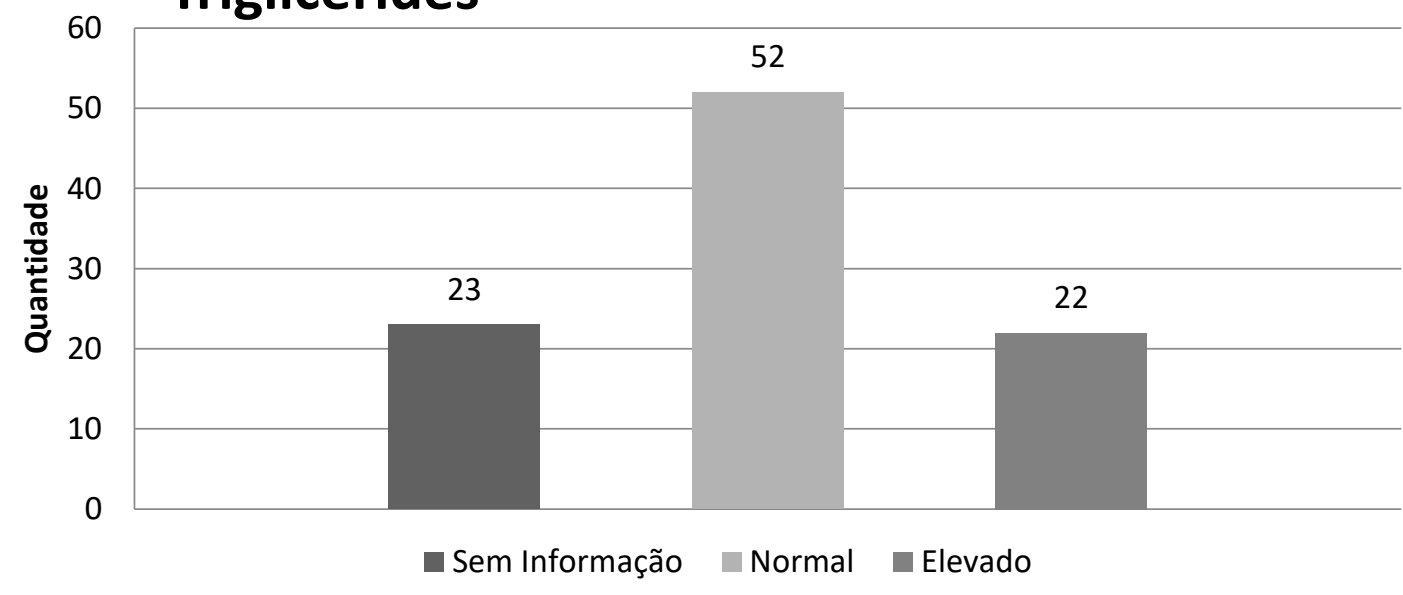

Fonte: Pesquisa própria,2016. 


\section{Discussão}

A presente pesquisa evidenciou que todos os jovens analisados possuem alimentação com consumo excessivo de carboidratos e a grande maioria deles ( mais de 91\%) com consumo excessivo de lipídeos (tabela1). Sendo assim, o estudo evidenciou que o perfil lipídico não é influenciado apenas pelo alto consumo de carboidratos e lipídios, visto que não observamos diferenças significativas quando testamos essas variáveis frente a ter os níveis de colesterol aumentado ou não. Entretanto, uma alimentação excessiva em carboidratos e lipídeos associados ao sedentarismo, levou em nossa amostra, alterações significativas nos níveis de colesterol total, indicando associação positiva e estatisticamente significante $\left(. x^{2}=8,00\right.$, $\mathrm{P}=0,005)$

A redução da ingestão o carboidrato da dieta levam à uma menor ingestão calórica total e podem ser substituídas por proteínas, auxiliando na perda de peso, resultando em alterações metabólicas, melhorando as variáveis lipídicas e reduzindo níveis plasmáticos de TG, VLDL, colesterol total, LDL(COOK; KAVEY, 2011).

As dislipidemias em jovens são mais frequentes provavelmente pela mudança de hábitos alimentares associados à redução de atividades físicas(DĀNISHGĀH-I 'ULŪM-I PIZISHKĪ-I IȘFAHĀN.; KANEKAR, 2011; SPOSITO et al., 2007).O aumento do consumo de gordura está associado com a maior concentração plasmática de colesterol e a maior incidência de aterosclerose.A gordura saturadae os ácidos graxos trans são as principais causas alimentares de elevação dosníveis de colesterol no sangue. Está relacionada com o aumento do colesterol total, do LDL-C e triglicerídeos e com a redução do HDL-C (o colesterol saudável). A gordura saturada é normalmente encontrada na forma sólida e em produtos de origem animal como leite integral, creme de leite, manteiga, queijos gordurosos (provolone, parmesão, mussarela), banha, bacon, toucinho, gordura das carnes, pele das aves e dos peixes já as gorduras trans estão presentes nos sorvetes, chocolates, pães e bolachas recheadas, molhos para salada, maionese, cremes para sobremesas e óleos para frituraindustrial(FRANCA; ALVES, 2005).

Apesar disso, é fundamental recordar que a hipercolesterolemia, na maior parte dos casos, está associada à outras condições que contribuem para o acontecimento desses eventos, como a ocorrência do sobrepeso e da obesidade, que por sua vez, está relacionado a uma série de fatores ambientais associados ao estilo de vida, entre os quais estão incluídas a inatividade 
física e as mudanças negativas nos hábitos alimentares que pode estar significativamente associado à ingestão insuficiente de alimentos protetores e à ingestão moderada ou excessiva de alimentos de risco (PINTO, 2012). Alterações na dieta voltada para restrição de carboidratos simples com a eliminação exclusiva de bebidas açucaradas é muito eficiente e quando aliada a uma atividade física, constituem uma base para prevenção daobesidade (KAVEY, 2015; M; JP; $\mathrm{RD}, 2011)$

Nos últimos 40 anos, a freqüência e a gravidade da obesidade infantil tem crescido consideravelmente e a consequiência do aumento do índice de massa corporal na criança pode determinar um jovem ou adulto obeso levando à complicações como doença cardiovascular, diabetes tipo2, doença hepática, por isso, devemos enfatizar a intervenção precoce para prevenir o aparecimento da obesidade na infância (KRAUSS et al., 2006).

Assim, a detecção desses fatores que levam à dislipidemia pode contribuir para o uma mudança do estilo de vida com vista à promoção da saúde e, assim, evitar que milhares de jovens desenvolvam prematuramente doença arterial coronariana e outras complicações.

\section{Conclusão}

Os dados indiciaram que a dislipidemia é evidente em uma porção pequena, mas não menos importante de crianças e adolescentes, seja na alteração de um ou mais parâmetros.Percebemos então a relevância da investigação antecipada destes fatores para a prevenção de doenças cardiovasculares.O cuidado com a saúde nesta faixaetária é muito importante, já que disfunções geradas na infância e adolescência persistem ou, até mesmo, evoluem na vida adulta. Os estudos sobre dislipidemias emcrianças e adolescentes devem ser expandidos no Brasil e no Mundo, para a maior percepção do público sobre os danos ocasionados à vida adulta.

\section{Referências}

BRASILEIRA, S.; CARDIOLOGIA, @ BULLET ISSN-0066-. V Diretriz Brasileira de Disli-pidemias e Prevenção da Aterosclerose. v. 101, n. 1, 2013. 
COOK, S.; KAVEY, R. E. W. Dyslipidemiaand pediatric obesity. Pediatric Clinics of North America, 2011. Disponível em: <https://www.ncbi.nlm.nih.gov/pmc/articles/PMC 3220879 /pdf/nihms323682.pdf>. Acesso em: 18 maio. 2017

DĀNISHGĀH-I 'ULŪM-I PIZISHKİ-I IȘFAHĀN., S.; KANEKAR, A. S. International journal of preventive medicine. [s.1.] Isfahan University of Medical Sciences, 2011. v. 3

EILAND LS et al. Use of statins for dyslipidemia in thepediatricpopulation. The journal of pediatric pharmacology and therapeutics : JPPT : theofficialjournalof PPAG, v. 15, n. 3, p. 160-172, 2010.

ENES, C. C.; SLATER, B. Obesidade na adolescência e seus principais fatores determinantes. Revista Brasileira de Epidemiologia, v. 13, n. 1, p. 163-171, mar. 2010.

FRANCA, E.; ALVES, J. G. B. Dislipidemia entre crianças e adolescentes de Pernambuco. Arquivo Brasileiro de Cardiologia, v. 87, n. 6, p. 722-727, 2005.

INSTITUTO BRASILEIRO DE GEOGRAFIA E ESTATÍSTICA-IBGE. Cidades@: Vitória da Conquista: Dados gerais do município, 2013. Disponível em: <http://www.ibge.gov.br /home/estatistica/ecnomia/comercioeservico/pas/pas2006>. Arquivo consultado em $15 \mathrm{de}$ maio de 2017.

KAVEY, R. E. W. Combined dys lipidemia in childhood. Journal of Clinical Lipidology, v. 9, n. 5, p. S41-S56, 2015.

KELSEY, M. M. et al. Age-related consequences of childhood obesity. Gerontology, v. 60, n. 3, p. 222-8, 2014.

KRAUSS, R. M. et al. Separate effect sofreduced carbohydratein take and weight lossonatherogenic dys lipidemia. The American journal of clinical nutrition, v. 83, n. 5, p. 1025-31; quiz 1205, maio 2006.

M, P.; JP, C.; RD, G. Obesity and physica lactivity in children. Canadian Family Physician, v. 57, n. 7, p. 779-782 4p, 2011.

NOGUEIRA, F.; CRISTINA CORRÊA DE SOUZA, M. Prevalência de Dislpidemias em Crianças e Adolescentes: Revisão Sistemática. Dislpidemias Prevalence In Children And Adolescents: A Systematic Review. Interbio, v. 8 n 2, p. 50-59, 2014.

PINTO, E. D. J. Fatores associados à dislipidemia em crianças e adolescentes de escolas públicas de Salvador, Bahia Factors associated with dyslipidemia in children and adolescents enrolled in public schools of Salvador, Bahia. RevBrasEpidemiol, v. 15, n. 2, p. 335-345, 2012.

RAMOS, A. T. et al. Lipid profile in overweight children an dadolescents. Journal of Human Growthand Development, v. 21, n. 3, p. 780-788, 2011. 
SERVICES, H. et al. Number 140 LipidScreening in Childhood for Detection of Multifac-torial Dyslipidemia : A Systematic Evidence Review for the U. S. Preventive Services Task Force. n. 140, 2012.

SPOSITO, A. C. et al. IV Diretriz Brasileira sobre Dislipidemias e Prevenção da Aterosclerose: Departamento de Aterosclerose da Sociedade Brasileira de Cardiologia. Arquivos Brasileiros de Cardiologia, v. 88, p. 2-19, abr. 2007.

UREK, R.; CRNCEVIĆ-UREK, M.; CUBRILO-TUREK, M. [Obesity--a global ublichealth problem]. Acta medica Croatica : asopisHravatskeakademijemedicinskihznanosti, v. 61, n. 2, p. 161-4, abr. 2007.

\section{Como citar este artigo (Formato ABNT):}

SAMPAIO, Sara C.; SOUSA, Beatriz R.; OLIVEIRA, Diana C.; ANDRADE, Adna G.F.; DUARTE, Stênio F. P. Perfil Lipídico de Jovens Escolares entre 2 e 19 anos no Interior da Bahia. Id on Line Revista Multidisciplinar e de Psicologia, Maio de 2017, vol.11, n.35, p.290-300. ISSN: 1981-1179.

Recebido: 23.05 .2017

Aceito: .24.05.2017 